\title{
Trends in In-Hospital Mortality among Patients with Stroke in China
}

\author{
Qian $\mathrm{He}^{19}$, Cheng $\mathrm{Wu}^{19}$, Hong Luo ${ }^{29}$, Zhi-Yong Wang ${ }^{3}$, Xiu-Qiang Ma ${ }^{1}$, Yan-Fang Zhao ${ }^{1}$, Jian Lu' ${ }^{1}$ \\ Chun Xiang ${ }^{1}$, Ying-Yi Qin ${ }^{1}$, Shun-Quan $\mathrm{Wu}^{1}$, Fei-Fei $\mathrm{Yu}^{1}$, Jia $\mathrm{He}^{1 *}$
}

1 Department of Health Statistics, Second Military Medical University, Shanghai, China, 2 Center of Educational Technology, Second Military Medical University, Shanghai, China, 3 Department of Information, Changhai Hospital, Second Military Medical University, Shanghai, China

\begin{abstract}
Background: The incidence and burden of stroke in China is increasing rapidly. However, little is known about trends in mortality during stroke hospitalization. The objectives of this study were to assess trends of in-hospital mortality among patients with stroke and explore influence factors of in-hospital death after stroke in China.

Methods: 109 grade III class A hospitals were sampled by multistage stratified cluster sampling. All patients admitted to hospitals between 2007 and 2010 with a discharge diagnosis of stroke were included. Trends in in-hospital mortality among patients with stroke were assessed. Influence factors of in-hospital death after stroke were explored using multivariable logistic regression.

Results: Overall stroke hospitalizations increased from 79,894 in 2007 to 85,475 in 2010, and in-hospital mortality of stroke decreased from $3.16 \%$ to $2.30 \%(P<0.0001)$. The percentage of severe patients increased while odds of mortality $(2010$ versus 2007) decreased regardless of stroke type: subarachnoid hemorrhage (OR 0.792, 95\% $\mathrm{Cl}=0.636$ to 0.987 ), intracerebral hemorrhage (OR $0.647,95 \% \mathrm{Cl}=0.591$ to 0.708$)$, and ischemic stroke (OR $0.588,95 \% \mathrm{Cl}=0.532$ to 0.649 ). In multivariable analyses, older age, male, basic health insurance, multiple comorbidities and severity of disease were linked to higher odds of in-hospital mortality.

Conclusions: The mortality of stroke hospitalizations decreased likely reflecting advancements in stroke care and prevention. Decreasing of mortality with increasing of severe stroke patients indicated that we should pay more attention to rehabilitation and life quality of stroke patients. Specific individual and hospital-level characteristics may be targets for facilitating further declines.
\end{abstract}

Citation: He Q, Wu C, Luo H, Wang Z-Y, Ma X-Q, et al. (2014) Trends in In-Hospital Mortality among Patients with Stroke in China. PLoS ONE 9(3): e92763. doi:10.1371/journal.pone.0092763

Editor: Alice Y. W. Chang, Kaohsiung Chang Gung Memorial Hospital, Taiwan

Received August 9, 2013; Accepted February 26, 2014; Published March 20, 2014

Copyright: ( $\odot 2014 \mathrm{He}$ et al. This is an open-access article distributed under the terms of the Creative Commons Attribution License, which permits unrestricted use, distribution, and reproduction in any medium, provided the original author and source are credited.

Funding: This study was funded by the key discipline construction of Evidence-based Public Health in Shanghai No 12GWZX0602, 2 national "Eleven Five" "Significant new drugs creation" special science and technology major (2008ZX09312-007, 2009ZX09312-025), the foundation of Faculty of Health Service, Second Military Medical University (2013wk02), the foundation of Second Military Medical University (2012JS11). The funders had no role in study design, data collection and analysis, decision to publish, or preparation of the manuscript.

Competing Interests: The authors have declared that no competing interests exist.

*E-mail: hejia63@yeah.net

9 These authors contributed equally to this work.

\section{Introduction}

Stroke is one of the leading causes of death and disability throughout the world [1], responsible for 4.4 million (9\%) of the total 50.5 million deaths each year [2]. The incidence and burden of stroke in China is increasing rapidly over time just like in other developing countries. About 2 million people of all ages suffer a new stroke, and 15 million stroke-related deaths occur in each year [3]. It is now becoming the first leading cause of death in China [1]. Mortality of stroke at discharge significantly increased with age, with $1.15 \%, 1.46 \%, 3.31 \%$, and $7.63 \%$ in-hospital mortality according to age group $(\leq 45,46-65,66-79, \geq 80$ years old) respectively, and the very old patients had the worst outcomes even after adjusted by prognostic factors[4]. Now, demographic ageing is occurring at an unprecedented rate worldwide; the proportion of Chinese aged 65 and over will increase from $4 \%$ in
2000 to $14 \%$ by 2025 , amounting to 200 million old people [5]. Aging may also result in an increased risk for stroke [6], which produces more and more burden on society and families [1]. It becomes very urgent to understand the trend of stroke prognosis. However, recent information about in-hospital mortality trends after stroke hospitalization is lack in China, though historical and risk factors of mortality after stroke have been identified in various studies including clinical trials, community-based studies, and voluntary registries[4,7]. With population aging, the health reform was deepened and stroke prevention, treatment and rehabilitation care were promoted continually. Knowledge of in-hospital deaths after stroke may be helpful for knowing the "real-world" and interface of challenges in optimizing overall premorbid health status, stroke prevention, acute stroke treatment, and acute general medical care at the individual, hospital, and health system levels [7]. 
Analyzing of influence factors of trend of stroke outcomes may lead to strategies for enhancing quality improvement at several levels of policymaking [7]. The objectives of this study were to assess trends of the proportion of stroke hospitalizations that resulted in death in China in recent years and find some potential influence factors of in-hospital mortality after stroke hospitalization.

\section{Methods}

\section{Setting, sampling and study design}

Using a multistage stratified cluster sampling method, 109 grade III (third tier) class A hospitals were enrolled representing 765 grade III class A hospitals in China. They were from the northern (10 provinces), eastern (4 provinces), southern (7 provinces), western (10 provinces) China respectively. The hospitals in China were classed to 9 types: grade III class A, grade III class B, grade III class C, grade II class A, grade II class B, grade II class C, grade I class A, grade I class B, and grade I class $\mathrm{C}$. The highest rank is the Grade III class A hospital, which is a large-scale general hospital integrating medical service, education and research including more than 500 beds. From 2007 to 2010, the discharges from sampled hospitals for the calendar year were selected into this study.

\section{Data collection and analysis}

To identify stroke hospitalizations according to the primary diagnosis, we used all discharges for which International Classification of Diseases, 9th Revision (ICD-9) codes 430 to 438 in primary diagnosis for conservative estimation [7,8]. When analyzing based on hospital diagnosis statistics, generally they count cases, not patients [7]. A person treated several times at hospital will be counted multiply. 350036 discharges that had stroke from January 2007 to December 2010 were recruited.

Data were captured including demographics, discharge-level information on diagnoses, primary payer (self-payment, basic health insurance, other), comorbid condition, severity of disease (mild, moderate and severe), results of treatment, length of stay in hospital (LOS) and hospital region (northern, eastern, southern and western China). We considered all discharges to be independent.

The study was approved by the Ethics Committee of the Second Military Medical University, Shanghai, China. The ethics committee waived the need of informed consent for the study because of its retrospective nature and data were analyzed anonymously.

\section{Statistical Analysis}

Comparing baseline characteristics between 2007 and 2010, numerical variables which followed normal distribution were analyzed by ANOVA, non-normal distribution were analyzed by Kruskal-Wallis test, such as age and LOS. Unordered categorical variables were analyzed by chi-square test or Fisher's exact test, including gender, primary payer, hospital region; ordinal categorical variables were analyzed by Kruskal-Wallis test including Charlson comorbidity index (CCI) and severity of disease.

Trends of in-hospital mortality after stroke were analyzed across time stratified by stroke type and hospital region. Stroke type was categorized as follows [6,7,9]: (1) subarachnoid hemorrhage (SAH; ICD-9 430); (2) intracerebral hemorrhage (ICH; ICD-9 431); (3) ischemic stroke (IS; ICD-9 433, 434, and 436). To assess linear trend of in-hospital mortality from year 2007 to 2010, the Cochran-Armitage trend test was used. To assess adjusted inhospital mortality trends and identify independent predictors of in- hospital mortality, the multivariable logistic regression model was used. The following demographic and clinical characteristics were adjusted: age, gender, primary payer, comorbid condition, severity of disease, and LOS. In addition, the hospital region was also adjusted for. Year was involved as a categorical variable which was transformed to dummy variables in the model.

The severity of disease was classified into three groups by National Institutes of Health Stroke Scale (NIHSS), and they were mild $(\leq 6)$, moderate $(\geq 7$ and $\leq 14)$ and severe $(\geq 15)$. The number and severity of comorbid conditions were assessed using the Charlson comorbidity index (CCI). The modified version of the CCI was used ${ }^{7}$. The CGI is a weighted score composed of 17 comorbid conditions including congestive heart failure (weight 1), myocardial infarction (weight 1), chronic pulmonary disease (weight 1), cerebrovascular disease (weight 1), hemiplegia or paraplegia (weight 2), dementia (weight 1), diabetes without complications (weight 1), diabetes with complication (weight 2), malignancy (weight 2), metastatic solid tumor (weight 6), mild liver disease (weight 1), moderate or severe liver disease (weight 3), peptic ulcer disease (weight 1), peripheral vascular disease (weight 1), rheumatologic disease (weight 1), renal disease (weight 2), and AIDS (weight 6). In the multivariable analysis, CGI was grouped into 4 categories [7], including a CGI of $1,2,3$, or $\geq 4$.

All tests were 2-tailed, and $P \leq 0.05$ was considered significant. Statistical analysis was performed using a commercially available software package (SAS statistical software version 9.3; SAS Institute Inc., Cary, NC, USA).

\section{Results}

Overall, stroke hospitalizations increased from 79894 in 2007 to 85475 in 2010 in the 109 grade III class A hospitals, whereas overall percentage of stroke hospitalizations that resulted in death decreased from $3.16 \%$ to $2.30 \%(P<0.0001)$. Over these years, the mortality of stroke inpatients declined steadily, a trend generally seen across stroke types (Figure 1). Table 1 shows the summary for demographic, clinical, and regional factors for each stroke types. From 2007 to 2010, there was a modest but significant increase in age for SAH $(P=0.0054)$ and IS $(P<0.0001)$, but not for ICH $(P=0.2572)$. And compared with 2007 , there were more males in 2010 for IS $(P=0.0211)$ but not for SAH or ICH $(P=0.8438$ or $0.2562)$. During these years, primary payer changed significantly for all stroke types $(P<0.0001)$, and there were more patients who paid by basic health insurance while fewer self-payment. The CGI became less by year $(P<0.0001)$, and the proportion of severe status was increased $(P<0.0001)$. The number of inpatient increased in north for SAH $(P<0.0001)$, west for $\mathrm{ICH}$ $(P<0.0001)$, south and west for IS $(P<0.0001)$. The LOS of IS decreased $(P<0.0001)$ while ICH increased $(P=0.0010)$, and the change of SAH was not significant.

From 2007 to 2010, SAH, ICH and IS hospitalizations increased by $15.32 \%, 4.75 \%$ and $18.80 \%$ respectively. In-hospital mortality rates across time for each stroke types are shown in Table 2 and Figure 1. There was a decrease in the in-hospital mortality of SAH (from $6.28 \%$ to $5.04 \% ; P=0.0167$ ), ICH (from $9.73 \%$ to $6.52 \% ; P<0.0001$ ) and IS (from $2.48 \%$ to $1.47 \%$; $P<0.0001)$. There were similar trend in northern, eastern, southern and western China for ICH and IS (Figure S1). But for SAH mortality decreased significantly in southern and western China, while the mortality in north was the highest. Comparisons of the decrease in in-hospital mortality across time among the different types of stroke are listed in Table 3 (ORs for the effect of year). 


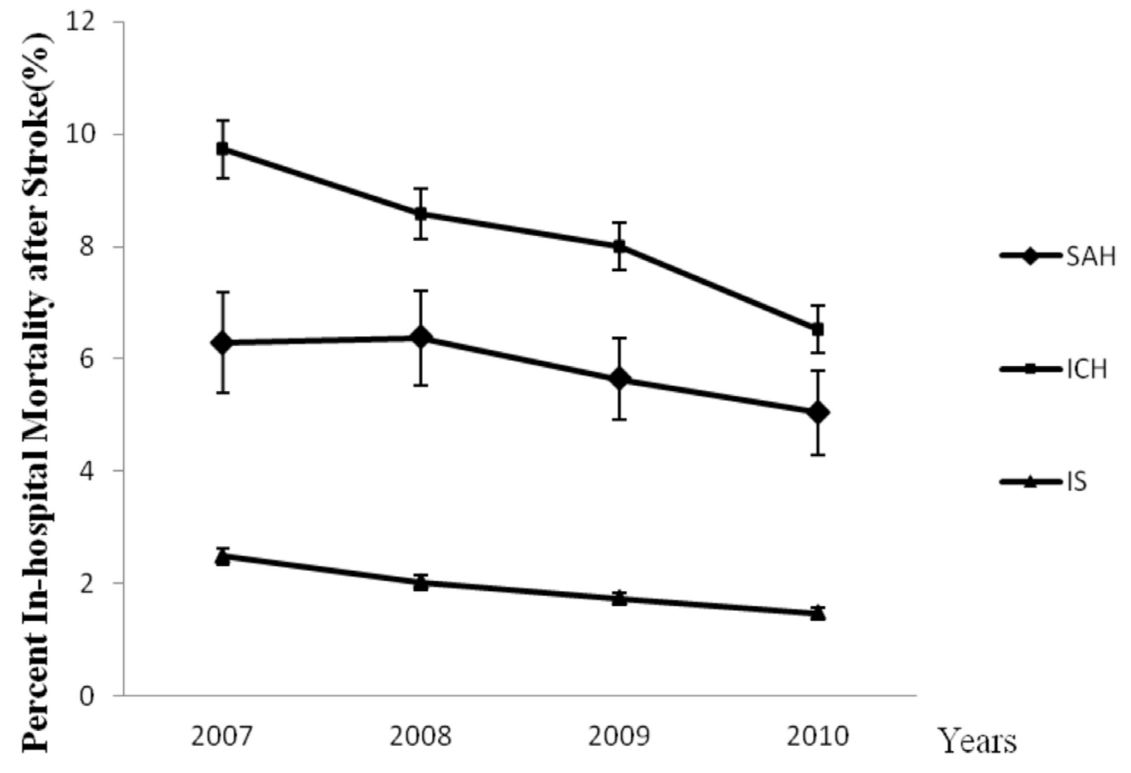

Figure 1. In-Hospital Mortality after Stroke by Type between 2007 and 2010 in China. doi:10.1371/journal.pone.0092763.g001

Table 4 shows the adjusted logistic regression analyses for each stroke type. For hospitalizations with $\mathrm{SAH}$ or ICH, older age, basic health insurance payment (versus self-pay), higher CCI, severe status, hospitals located in the northern and southern region (versus western) were significantly associated with higher odds ratios of dying in the hospital, whereas later year, female and LOS were associated with a significant decrease in the risks of inhospital death. For hospitalizations with IS, older age, basic health insurance (versus self-pay), higher CCI, severe status, hospitals located in the northern region (versus western) were significantly associated with increasing in the risks of dying in the hospital, while later years, and LOS were associated with a significant decrease in the risks of in-hospital mortality. The OR estimates for the effect of year were unaltered after including covariates in the models.

\section{Discussion}

This analysis of proportions of in-hospital mortality after stroke from 2007 to 2010 in China showed that deaths during stroke hospitalization have lessened significantly over time and since 2008 the decline has been steady and continuous, probably mainly reflecting improvements in hospital care after occurrence of a stroke. The trend of decrease in the percentage of stroke hospitalizations resulting in death is consistent with the observational study from the Sino-MONICA-Beijing from 1984 to 2004 [10] and the crude mortality of cerebrovascular disease from the fourth health service survey in China [11]. The proportion of IS subtype were the largest, followed by ICH and SAH in the hospitals, and these data are generally in accordance with the incidence of stroke in the survey based on community population and the China National Stroke Registry (CNSR) from 2003-2008 in China [12-15]. And the proportion of male was higher than female in IS and ICH,which was similar with other studies $[7,12,15]$, while the proportion of female was higher than male in SAH, which was similar with Beijing and Shanghai in China from 1991-2000 [16]. The median age was 68 years old among IS patients while less than 60 years old among ICH and SAH patients, and age difference among subtypes also appeared in some community population [13]. There was a good representativeness for stroke patients because of the similar distribution of subtype, gender and age with the observational studies based on community population and CNSR. This study showed that the mortality decreased from $3.16 \%$ in 2007 to $2.30 \%$ in 2010 (SAH: form $6.28 \%$ to $5.04 \%$, ICH: $9.73 \%$ to $6.52 \%$, IS: from 2.48 to $1.47 \%$ ) while the number of patients increased from 79894 to 85 475. The decrease trend was also found in the United States $\mathrm{SAH}$ : from $26.90 \%$ in $1997-1998$ to $23.80 \%$ in $2005-2006$, ICH: from $30.47 \%$ to $28.23 \%$, IS: from $9.76 \%$ to $8.78 \%$ ) [7] and in Germany (from $11.9 \%$ in 2005 to $9.5 \%$ in 2010) [17]. The in-hospital mortality in this study was nearly equal to the data from the China National Registry [18], but lower than Germany and the United States. There may be some reasons. Firstly, the age of patients in this study was younger (this study: 65 vs. Germany: 73 years old). Secondly, the proportion of $\mathrm{ICC} \geq 4$ which reflects severe level of stroke was very low compared to America (this study in 2007 vs America in 2005-2006: SAH 0.25 vs 10.2 , ICH 0.65 vs 24.3 , IS 1.44 vs 29.4). Thirdly, patients were earlier transferred to smaller regional hospitals or home. Fourthly, repetitive admission would decrease the mortality. The decreasing of mortality in the United States was likely driven by revascularization strategies among ischemic strokes and better acute stroke care in addition to prevention of stroke [7]. Some health reform strategy and mass approaches on decreasing mortality were also adopted in China including stroke prevention, various unconventional local therapeutic traditions, and several national guidelines on stroke prevention and treatment $[11,19,20]$.

On the other hand, the construction of health insurance system was accelerated under the health reform policy and the proportion of basic health insurance became higher through these years. It helped with decreasing catastrophic healthcare payments and more stroke patients gained positive treatment [21]. Severity and CCI were the risk factors for stroke [7]. There were more patients in the northern and western regions. For the different stroke types, 
Table 1. Descriptive Socio-demographic and Clinical Characteristics of Hospitalized Patients in China with a Diagnosis of Stroke between 2007 and 2010 by Stroke Type.

\begin{tabular}{|c|c|c|c|c|c|c|c|c|c|c|c|c|}
\hline \multirow[b]{2}{*}{ Variables } & \multicolumn{4}{|l|}{ SAH } & \multicolumn{4}{|l|}{ ICH } & \multicolumn{4}{|l|}{ IS } \\
\hline & \multicolumn{2}{|l|}{2007} & \multicolumn{2}{|c|}{2010} & \multicolumn{2}{|l|}{2007} & \multicolumn{2}{|l|}{2010} & \multicolumn{2}{|l|}{2007} & \multicolumn{2}{|l|}{2010} \\
\hline & \multicolumn{2}{|c|}{$(N=2787)$} & \multicolumn{2}{|c|}{$(N=3214)$} & \multicolumn{2}{|c|}{$(N=12841)$} & \multicolumn{2}{|c|}{$(N=13451)$} & \multicolumn{2}{|c|}{$(N=38418)$} & \multicolumn{2}{|c|}{$(N=45641)$} \\
\hline Age, years & 53 & $43-62$ & 54 & $45-63$ & 58 & $49-69$ & 58 & $49-69$ & 68 & $57-75$ & 68 & $58-76$ \\
\hline Median, $Q_{1}-Q_{3}$ & 53 & $43-62$ & 54 & $45-63$ & 58 & $49-69$ & 58 & $49-69$ & 68 & $57-75$ & 68 & $58-76$ \\
\hline$<45$ & 803 & 28.82 & 761 & 23.68 & 2142 & 16.68 & 2060 & 15.31 & 2163 & 5.63 & 2161 & 4.73 \\
\hline $45-64$ & 1397 & 50.14 & 1757 & 54.67 & 6143 & 47.84 & 6709 & 49.88 & 13629 & 35.48 & 17132 & 37.57 \\
\hline $65-74$ & 381 & 13.68 & 444 & 13.81 & 2760 & 21.49 & 2624 & 19.51 & 12358 & 32.17 & 13141 & 28.79 \\
\hline $75-79$ & 120 & 4.31 & 147 & 4.57 & 999 & 7.78 & 1151 & 8.56 & 5566 & 14.49 & 6905 & 15.13 \\
\hline 80 & 85 & 3.05 & 105 & 3.27 & 797 & 6.21 & 907 & 6.74 & 4702 & 12.24 & 6302 & 13.81 \\
\hline \multicolumn{13}{|l|}{ Sex } \\
\hline Male & 1317 & 47.27 & 1506 & 46.86 & 8037 & 62.59 & 8539 & 63.48 & 23129 & 60.20 & 27557 & 60.38 \\
\hline Female & 1469 & 52.73 & 1708 & 53.14 & 4804 & 37.41 & 4912 & 36.52 & 15288 & 39.80 & 18084 & 39.62 \\
\hline \multicolumn{13}{|l|}{ Primary payer } \\
\hline self-payment & 1890 & 67.84 & 1659 & 51.62 & 8586 & 66.86 & 6802 & 50.57 & 19581 & 50.97 & 16601 & 36.37 \\
\hline basic health insurance & 704 & 25.27 & 1362 & 42.38 & 3718 & 28.95 & 6193 & 46.04 & 16674 & 43.40 & 27062 & 59.29 \\
\hline other & 192 & 6.89 & 193 & 6.00 & 537 & 4.19 & 456 & 3.40 & 2163 & 5.63 & 1978 & 4.34 \\
\hline \multicolumn{13}{|l|}{$\mathrm{CCl}$} \\
\hline 1 & 2431 & 87.29 & 2865 & 89.14 & 11064 & 86.16 & 12220 & 90.85 & 26790 & 69.73 & 35184 & 77.89 \\
\hline 2 & 312 & 11.20 & 320 & 9.96 & 1311 & 10.21 & 795 & 5.91 & 9761 & 25.41 & 9093 & 19.92 \\
\hline 3 & 35 & 1.26 & 26 & 0.81 & 383 & 2.98 & 364 & 2.71 & 1314 & 3.42 & 1019 & 2.23 \\
\hline$\geq 4$ & 7 & 0.25 & 3 & 0.09 & 83 & 0.65 & 72 & 0.53 & 553 & 1.44 & 345 & 0.76 \\
\hline \multicolumn{13}{|l|}{ Severity of disease } \\
\hline severe & 77 & 2.76 & 198 & 6.16 & 330 & 2.57 & 559 & 4.16 & 157 & 0.42 & 348 & 0.76 \\
\hline moderate & 1173 & 42.10 & 1451 & 45.15 & 5689 & 44.30 & 6021 & 44.76 & 10011 & 26.06 & 11547 & 25.30 \\
\hline mild & 1536 & 55.14 & 1565 & 48.69 & 6822 & 53.13 & 6871 & 51.08 & 28250 & 73.52 & 33746 & 73.94 \\
\hline \multicolumn{13}{|l|}{ Hospital region } \\
\hline North (10 provinces) & 950 & 34.10 & 1097 & 34.13 & 5446 & 42.41 & 5455 & 40.55 & 19486 & 50.72 & 22683 & 49.70 \\
\hline East (4 provinces) & 579 & 20.78 & 610 & 18.98 & 1776 & 13.83 & 2050 & 15.24 & 5191 & 13.51 & 5271 & 11.55 \\
\hline South (7 provinces) & 511 & 18.34 & 558 & 17.36 & 2166 & 16.87 & 2232 & 16.59 & 4976 & 12.95 & 6556 & 14.36 \\
\hline West (10 provinces) & 746 & 26.78 & 949 & 25.53 & 3453 & 26.89 & 3714 & 27.62 & 8765 & 22.82 & 11131 & 24.39 \\
\hline \multicolumn{13}{|l|}{ Length of stay } \\
\hline Days (Median, $Q_{1}-Q_{3}$ ) & 12 & $5-20$ & 12 & $5-19$ & 14 & $6-23$ & 15 & $7-24$ & 13 & $9-18$ & 12 & $8-16$ \\
\hline
\end{tabular}

doi:10.1371/journal.pone.0092763.t001

more SAH patients appeared in the north, more ICH in the west, and more IS in the south and west. It is partly due to different climates and lifestyles [20]. The mortality of all subtypes of stroke was higher in the north, though it decreased significantly except for SAH.

The change of LOS among SAH patients was no significant, and the LOS among ICH became longer while IS shorter. Mortality of ICH was the highest among all subtypes and needed more time to recover. The health insurance strategy limited the LOS in hospitals due to cost containment, and the IS patients would be transferred to other community hospitals for further recovery. The mortality of ICH was highest, followed by SAH and IS, and decreasing trend was significant in SAH and IS over the time. ORs for the effect of time 2010 versus 2007 on in-hospital mortality for each stroke subtype also showed that the percentage of stroke hospitalizations resulting in death decreased in China, similar with the United States [7].

The multivariable analyses investigated the independent influence factors of dying in the hospital after a stroke, and showed that several socio-demographics and region factors could be further explored to possibly boost survival among hospitalized patients. Many of these factors were similar across stroke types, which included older age, male, northern and western regions and which had previously been associated with larger risk of short-term mortality after stroke, and underscored the need to implement strategies to bridge these socio-demographic gaps in stroke care $[4,14,20]$. In this study, patients whose primary payer was the basic health insurance had more odds of mortality in hospital. 
Table 2. Distribution of In-Hospital Mortality Rates across Time by Stroke Type and Region.

\begin{tabular}{|c|c|c|c|c|c|c|}
\hline Diagnosis & Year & Overall (\%) & North (\%) & East (\%) & South (\%) & West (\%) \\
\hline \multicolumn{7}{|l|}{$\mathrm{SAH}$} \\
\hline & 2007 & 6.28 & 6.63 & 4.84 & 6.26 & 6.97 \\
\hline & 2008 & 6.37 & 7.56 & 4.68 & 7. 87 & 5.00 \\
\hline & 2009 & 5.64 & 7.03 & 4.27 & 5.90 & 4.75 \\
\hline & 2010 & 5.04 & 7.57 & 3.44 & 3.23 & 4.21 \\
\hline & Z statistic & -2.393 & 0.5808 & -1.2429 & -2.4706 & -2.445 \\
\hline & $P$ value & 0.0167 & 0.5614 & 0.2139 & 0.0135 & 0.0145 \\
\hline \multicolumn{7}{|l|}{$\mathrm{ICH}$} \\
\hline & 2007 & 9.73 & 10.98 & 9.29 & 9.51 & 8.14 \\
\hline & 2008 & 8.58 & 9.12 & 8.01 & 8.75 & 7.91 \\
\hline & 2009 & 8.00 & 8.99 & 6.23 & 8.89 & 6.93 \\
\hline & 2010 & 6.52 & 7.52 & 5.37 & 6.45 & 5.74 \\
\hline & Z statistic & -9.5578 & -5.9840 & -5.1693 & -3.3775 & -4.3044 \\
\hline & $P$ value & $<0.0001$ & $<0.0001$ & $<0.0001$ & 0.0007 & $<0.0001$ \\
\hline \multicolumn{7}{|l|}{ IS } \\
\hline & 2007 & 2.48 & 2.49 & 3.10 & 2.11 & 2.32 \\
\hline & 2008 & 2.02 & 2.02 & 2.15 & 1.97 & 1.98 \\
\hline & 2009 & 1.73 & 1.97 & 1.78 & 1.27 & 1.49 \\
\hline & 2010 & 1.47 & 1.66 & 1.84 & 1.19 & 1.10 \\
\hline & Z statistic & -11.1168 & -5.8082 & -4.6052 & -4.7562 & -7.2555 \\
\hline & $P$ value & $<0.0001$ & $<0.0001$ & $<0.0001$ & $<0.0001$ & $<0.0001$ \\
\hline \multicolumn{7}{|l|}{ Overall } \\
\hline & 2007 & 3.16 & 3.24 & 3.62 & 3.35 & 2.69 \\
\hline & 2008 & 2.94 & 2.91 & 3.09 & 3.36 & 2.67 \\
\hline & 2009 & 2.79 & 3.00 & 2.52 & 2.85 & 2.54 \\
\hline & 2010 & 2.30 & 2.50 & 2.38 & 2.12 & 2.03 \\
\hline & Z statistic & -10.6863 & -5.5016 & -6.0191 & -6.3088 & -4.4895 \\
\hline & $P$ value & $<0.0001$ & $<0.0001$ & $<0.0001$ & $<0.0001$ & $<0.0001$ \\
\hline
\end{tabular}

doi:10.1371/journal.pone.0092763.t002

Patients would choose treatment in hospital much more easily and not care the high expenditure if they had the basic health insurance. Generally patients in worse condition were more likely to die during stroke hospitalization. However, it was surprising to observe that the number of hospitalized stroke patients in server condition has risen over these years. It may be because that condition of patients are progressively getting worse, but a more likely explanation in the face of declining in-hospital mortality rates after stroke could be that better diagnostic techniques and better awareness have led to increased diagnoses of several general medical conditions over the years. Considering on decreasing of

Table 3. ORs for the Effect of Year on In-Hospital Mortality for Each Stroke Type.

\begin{tabular}{lll}
\hline & & \\
\hline Stroke Type & $\mathbf{O R}^{*}(\mathbf{9 5 \%} \mathrm{Cl})$ & $P$ value \\
\hline SAH & $0.792(0.636,0.987)$ & 0.0377 \\
ICH & $0.647(0.591,0.708)$ & $<0.0001$ \\
IS & $0.588(0.532,0.649)$ & $<0.0001$ \\
\hline
\end{tabular}

* odds ratio of 2010 versus 2007, taking year 2007 as the reference group doi:10.1371/journal.pone.0092763.t003 mortality and increasing of number of worse stroke patients, the rehabilitation and life quality of stroke patients should be paid more attentions in China. Since 2009 national screening, prevention and control of stroke project was promoted. The CGI became lower, which is inconsistent with the United State [7].

This study has limitations. Because of the hospitals were grade III class A, this analysis would not reflected other grade hospitals completely though the gender, age, subtype distribution were the same with the survey and community population. So earlier transfer of patients to smaller regional hospitals would bias the numbers of intra-hospital mortality.

Other limitations include inability to distinguish first from recurrent strokes, and lack of documented information on the rate of non-hospitalized stroke in the country because many strokerelated deaths occurred out of the hospital. The study was strengthened by its nationwide scope, incident and not prevalent stroke rate data, clinician-diagnosed and not self-reported strokes. The time-interval may be a little short in this study, further research should be taken. 
Table 4. Logistic Regression Analyses of Influence Factors of In-Hospital Mortality after Stroke from 2007 to 2010 in China.

\begin{tabular}{|c|c|c|c|c|c|c|}
\hline \multirow[b]{2}{*}{ Variables } & \multicolumn{2}{|l|}{ SAH } & \multicolumn{2}{|l|}{$\mathbf{I C H}$} & \multicolumn{2}{|l|}{ IS } \\
\hline & OR $(95 \% \mathrm{Cl})$ & $P$ value & OR $(95 \% \mathrm{Cl})$ & $P$ value & OR $(95 \% \mathrm{Cl})$ & $P$ value \\
\hline \multicolumn{7}{|l|}{ Years } \\
\hline 2008 vs. 2007 & $0.93(0.75,1.16)$ & 0.5350 & $0.83(0.76,0.90)$ & $<0.0001$ & $0.78(0.71,0.86)$ & $<0.0001$ \\
\hline 2009 vs. 2007 & $0.83(0.67,1.02)$ & 0.0795 & $0.74(0.68,0.81)$ & $<0.0001$ & $0.66(0.60,0.72)$ & $<0.0001$ \\
\hline 2010 vs. 2007 & $0.67(0.53,0.84)$ & 0.0006 & $0.59(0.54,0.65)$ & $<0.0001$ & $0.55(09.49,0.60)$ & $<0.0001$ \\
\hline \multicolumn{7}{|l|}{ Demographic factors } \\
\hline Female vs. Male & $0.81(0.69,0.94)$ & 0.0066 & $0.75(0.70,0.80)$ & $<0.0001$ & $1.01(0.94,1.08)$ & 0.8194 \\
\hline Age (per year) & $1.04(1.04,1.05)$ & $<0.0001$ & $1.02(1.01,1.02)$ & $<0.0001$ & $1.06(1.05,1.06)$ & $<0.0001$ \\
\hline \multicolumn{7}{|l|}{ Primary payer } \\
\hline $\begin{array}{l}\text { basic health insurance } \\
\text { vs. self-pay }\end{array}$ & $1.69(1.43,1.99)$ & $<0.0001$ & $1.82(1.70,1.94)$ & $<0.0001$ & $1.43(1.32,1.54)$ & $<0.0001$ \\
\hline other vs. self-pay & $0.88(0.61,1.27)$ & 0.4982 & $1.35(1.16,1.58)$ & 0.0001 & $1.31(1.11,1.54)$ & 0.0012 \\
\hline \multicolumn{7}{|l|}{$\mathrm{CCl}$} \\
\hline 2 vs. 1 & $2.68(0.97,7.34)$ & 0.0561 & $2.88(2.19,3.80)$ & $<0.0001$ & $3.00(2.43,3.70)$ & $<0.0001$ \\
\hline 3 vs. 1 & $2.60(1.56,4.34)$ & 0.0003 & $2.30(2.00,2.65)$ & $<0.0001$ & $2.54(2.22,2.90)$ & $<0.0001$ \\
\hline$\geq 4$ vs. 1 & $1.49(1.19,1.88)$ & 0.0007 & $1.25(1.13,1.39)$ & $<0.0001$ & $0.94(0.86,1.03)$ & 0.1753 \\
\hline \multicolumn{7}{|l|}{ Severity of disease } \\
\hline severe vs. mild & $1.69(1.20,2.37)$ & 0.0025 & $1.39(1.19,1.63)$ & $<0.0001$ & $5.06(3.95,6.50)$ & $<0.0001$ \\
\hline moderate vs. mild & $1.22(1.04,1.43)$ & 0.0171 & $1.25(1.17,1.33)$ & $<0.0001$ & $1.85(1.72,1.98)$ & $<0.0001$ \\
\hline \multicolumn{7}{|l|}{ Hospital region } \\
\hline North vs. west & $1.33(1.09,1.62)$ & 0.0055 & $1.40(1.30,1.52)$ & $<0.0001$ & $1.17(1.07,1.28)$ & 0.0005 \\
\hline East vs. west & $0.81(0.62,1.05)$ & 0.1188 & $1.02(0.92,1.14)$ & 0.6739 & $1.01(0.89,1.13)$ & 0.9310 \\
\hline South vs. west & $1.32(1.03,1.69)$ & 0.0269 & $1.41(1.28,1.56)$ & $<0.0001$ & $1.01(0.89,1.15)$ & 0.8639 \\
\hline Length of stay & $0.94(0.93,0.95)$ & $<0.0001$ & $0.94(0.94,0.94)$ & $<0.0001$ & $0.98(0.98,0.98)$ & $<0.0001$ \\
\hline
\end{tabular}

doi:10.1371/journal.pone.0092763.t004

\section{Supporting Information}

Figure S1 In-Hospital Mortality after Stroke by Type between 2007 and 2010 in the Different Regions. Data presented trends of in-hospital mortality and its 95\% CI in different years of three stroke types including SAH, ICH and IS. (TIF)

\section{References}

1. Wang Z, Li J, Wang C, Yao X, Zhao X, et al. (2013) Gender differences in 1year clinical characteristics and outcomes after stroke: Results from the China national stroke registry. PloS One 8: e56459.

2. Kissela BM, Khoury JC, Alwell K, Moomaw CJ, Woo D, et al. (2012) Age at stroke: Temporal trends in stroke incidence in a large, biracial population. Neurology 79: 1781-1787.

3. Cole JW, Naj AC, O’Connell JR, Stine OC, Sorkin JD, et al. (2007) Neuroserpin polymorphisms and stroke risk in a biracial population: The stroke prevention in young women study. BMC Neurol 7: 37.

4. Deng YX, Wang YL, Gao BQ, Wang CX, Zhao XQ et al. (2012) Age differences in clinical characteristics, health care, and outcomes after ischemic stroke in China. CNS Neurosci Ther 18: 819-826.

5. Liu ZR, Albanese E, Li S, Huang YQ, Ferri CP, et al. (2009) Chronic disease prevalence and care among the elderly in urban and rural Beijing, China - a 10/66 dementia research group cross-sectional survey. BMC Public Health 9: $394-405$.

6. Lichtman JH, Jones SB, Leifheit-Limson EC, Wang Y, Goldstein LB (2011) 30day mortality and readmission after hemorrhagic stroke among medicare beneficiaries in joint commission primary stroke center-certified and noncertified hospitals. Stroke 42: 3387-3391.

7. Ovbiagele B (2010) Nationwide trends in in-hospital mortality among patients with stroke. Stroke 41: 1748-1754.

\section{Acknowledgments}

We would like to thank Prof. Hong Meng for her considerate advices during the study.

\section{Author Contributions}

Conceived and designed the experiments: JH QH CW XQM. Performed the experiments: QH CW HL ZYW FFY. Analyzed the data: CX YYQ SQW. Wrote the paper: QH GW YFZ JL.

8. Kissela B, Schneider A, Kleindorfer D, Khoury J, Miller R, et al. (2004) Stroke in a biracial population: The excess burden of stroke among blacks. Stroke 35: $426-431$.

9. Lichtman JH, Jones SB, Wang Y, Leifheit-Limson EC, Goldstein LB (2013) Seasonal variation in 30-day mortality after stroke: Teaching versus nonteaching hospitals. Stroke 44: 531-533.

10. Zhao D, Liu J, Wang W, Zeng Z, Cheng J, et al. (2008) Epidemiological transition of stroke in China: Twenty-one-year observational study from the Sino-Monica-Beijing Project. Stroke 39: 1668-1674.

11. China's health statistics yearbook 2010. China's Ministry of Health web site. http://www.stats.gov.cn/tjsj/ndsj/. Accessed May 8, 2013.

12. Zhao Y, Zhao W, Cidan Z, Tashi C, Chun H, et al. (2011) An epidemiological survey of prevalence of stroke in urban and rural workers of Lhasa region. Chin J Neuromed 10: 1255-1258.

13. Rao Y, Chi H, Huang W, Huang L, Liu X, et al. (2007) Distribution characteristics of types of stroke in urban area in Shenzhen]. Xiandai Yufang Yixue 34: 1610-1611,1615.

14. Zhang LF, Yang J, Hong Z, Yuan GG, Zhou BF, et al. (2003) Proportion of different subtypes of stroke in China. Stroke 34: 2091-2096.

15. Wang Y, Cui L, Ji X, Dong Q Zeng J, et al. (2011) The China National Stroke Registry for patients with acute cerebrovascular events: Design, rationale, and baseline patient characteristics. Int J Stroke 6: 355-361. 
16. Jiang B, Wang WZ, Chen H, Hong Z, Yang QD, et al. (2006) Incidence and trends of stroke and its subtypes in China: Results from three large cities. Stroke 37: 63-68.

17. Nimptsch U, Mansky T (2012) Trends in Acute Inpatient Stroke Care in Germany An Observational Study Using Administrative Hospital Data From 2005-2010. Dtsch Arztebl Int 109(51-52): 885-892.

18. Wang PL, Zhao XQ, Yang ZH, Wang AX, Wang CX, et al. (2012) Effect of inhospital medical complications on case fatality post-acute ischemic stroke: data from the China National Stroke Registry. Chin Med J (Engl) 125(14): 24492454 .
19. Wang DZ, Gu Q Jiang GH, Yang DY, Zhang H, et al. (2012) [Time-series analysis on effect of air pollution on stroke mortality in Tianjin, China]. Zhonghua Lao Dong Wei Sheng Zhi Ye Bing Za Zhi 30: 902-907.

20. Liu M, Wu B, Wang WZ, Lee LM, Zhang SH, et al. (2007) Stroke in China: Epidemiology, prevention, and management strategies. Lancet Neurol 6: 456464.

21. Heeley E, Anderson CS, Huang Y, Jan S, Li Y, et al. (2009) Role of health insurance in averting economic hardship in families after acute stroke in China. Stroke 40: 2149-2156. 